\title{
Fractal Approach to Passivated Surface of Stainless Steel
}

\author{
Jung-Ho Heo ${ }^{a}$, Yong-Heon Lee ${ }^{b}$, Heon-Cheol Shin ${ }^{a^{*}}$ \\ ${ }^{a}$ School of Materials Science and Engineering, Pusan National University, Busan 609-735, Korea \\ bStainless Steel Research Group, POSCO, Pohang 790-785, Korea
}

(Received January 28, 2010 ; revised February 26, 2010 ; accepted February 27, 2010)

\begin{abstract}
The morphology of the passivated surface of stainless steel (SS) was quantitatively characterized based on fractal geometry. In particular, the surface irregularities of the passivated 304 and 439 SSs were comparatively analyzed in terms of their self-similar fractal dimensions. The passivated surface of 439 SS in an acid-based electrolyte proved to have a higher fractal dimension, as compared to that of $304 \mathrm{SS}$, esp. at a scale of several tens of nanometers, strongly indicating the higher irregularity of the passivated surface. It is anticipated that the fractal approach suggested herein might be effectively utilized to analyze the irregularity of the steel surface and/or the compactness of the oxide film.
\end{abstract}

Keywords: Corrosion, Passive film, Surface morphology, Fractal dimension, Stainless steel

\section{Introduction}

The porosity or structural irregularity of the passivated surface of stainless steel (SS) is often considered as a measure of its resistance to corrosion in an aggressive environment ${ }^{1,2)}$. Although there have been a number of studies on the structure, composition and atomic arrangement of the passive oxide film ${ }^{3-7)}$, in-depth quantitative studies on its morphology have been quite lacking, mostly because the usual quantitative methods of characterizing rough surfaces are quite limited (i.e., the rms roughness is exclusively used for the analysis of the surface morphology) and not very informative.

Following Mandelbrot's pioneering works on the scaling properties of objects and physical phenomena in nature ${ }^{8,9)}$, fractal geometry has been used in a number of research areas. Especially, rough and irregular surfaces have been frequently modeled as fractal and the correlation of the fractality with particular materials properties has been widely investigated $^{10-13)}$. In this work, we present a quantitative way to characterize the surface morphology of the

\footnotetext{
*Corresponding author. E-mail : hcshin@pusan.ac.kr
}

passive film formed on SS on the basis of fractal geometry. The fractal dimensions of the oxide films formed on type 304 and 439 SSs in acid-based solution are estimated at different scales and are discussed in terms of the surface irregularity and the passivity of the oxide film.

\section{Experimental Details}

Standard 304 and 439 SSs (POSCO, Republic of Korea) were used for this study. The specimens were successively polished with 240,600 and $1200 \mathrm{SiC}$ paper and finished with a $1 \mu \mathrm{m}$ diamond suspension to a mirror-like surface aspect. Then, they were rinsed with purified water in a sonicating bath. The electrochemical measurements were carried out with an electrochemical flat cell (EG\&G Model K0235) on the as-polished 304 and $439 \mathrm{SSs}$ in aqueous solutions of $5 \mathrm{wt} \% \mathrm{H}_{2} \mathrm{SO}_{4}$ and $5 \mathrm{wt} \% \mathrm{H}_{2} \mathrm{SO}_{4}+3.5 \mathrm{wt} \%$ $\mathrm{NaCl}$, previously deaerated with purified nitrogen gas for $24 \mathrm{~h}$. A platinum mesh and a saturated calomel electrode (SCE) were used as the counter and reference electrodes, respectively. In order to form the passive oxide films, the electrode potential was rapidly increased from the corrosion potential $\left(E_{\text {corr }}\right)$ to 

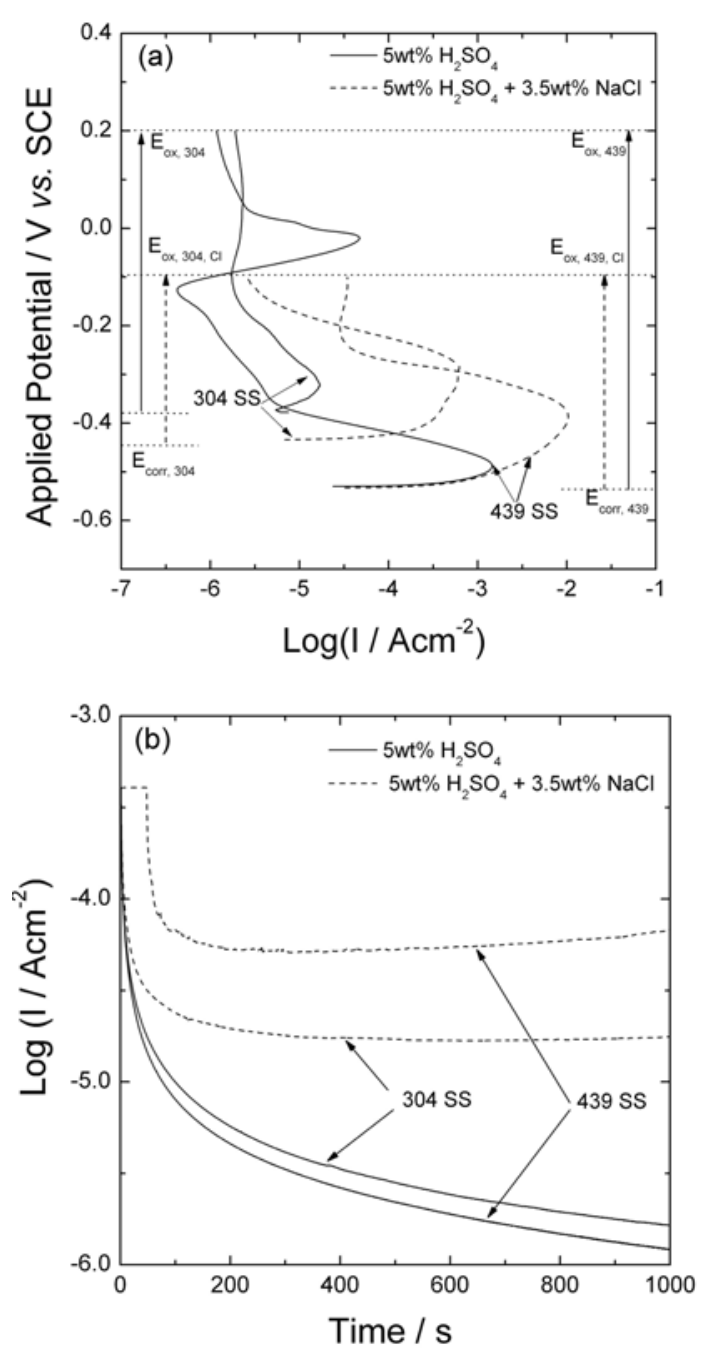

Fig. 1. (a) Potentiodynamic polarization curves of 304 and 439 SS measured in the aqueous solutions of $5 \mathrm{wt} \% \mathrm{H}_{2} \mathrm{SO}_{4}$ (solid line) and $5 \mathrm{wt} \% \mathrm{H}_{2} \mathrm{SO}_{4}+$ $3.5 \mathrm{wt} \% \mathrm{NaCl}$ (dashed line) and (b) current transients obtained by the potential jumps from corrosion potentials $E_{\text {corr }}$ to passivation potentials $E_{o x}$, as indicated in (a).

specific values $\left(E_{o x}\right)$ (Fig. 1(a)) and then maintained at $E_{o x}$ for $1000 \mathrm{~s}$ to ensure that the electrode was in a steady state condition (Fig. 1(b)).

The as-polished and as-oxidized samples were vacuum-sealed immediately after drying under nitrogen gas flow, in order to minimize the oxide formation in the air. The surface relief of the samples was observed using atomic force microscopy (AFM, XE120, Park Systems). The surface was scanned over areas of $0.5 \times 0.5 \mu \mathrm{m}^{2}$ with a resolution of $256 \times 256$ pixels. In order to check the self-similar scaling properties and estimate the fractal dimension $\left(D_{f}\right)$, the AFM images were analyzed by the triangulation method $^{14,15)}$ : the square flat plane $(x, y)$ with an area of $\mathrm{L}^{2}$ was divided into $\mathrm{N}^{2}$ equal segments, and the rough surface was covered by $2 \mathrm{~N}^{2}$ triangles with a projected size of $L_{p s}(=\mathrm{L} / \mathrm{N})$. The scaled surface area of the surface $A_{s c}$ was calculated by the summation of the areas of the $2 \mathrm{~N}^{2}$ triangles. Then, the $D_{f}$ value of the surface was given by $2-d \ln A_{s c} / d \ln L_{p s}$.

\section{Results and Discussion}

Shown in Figs. 2(a) and (b) are the AFM images of the as-polished samples of 304 and 439 SS, respectively. The fractal dimension of the surface was estimated with the help of the triangulation method (Fig. 3(a)) and summarized in Table 1. As shown in Fig. 3(a), there were roughly three regions having different scaling properties. That is, the aspolished surfaces have the highest fractal dimension, i.e., the most severe irregularity, of the order of less than $c a .30 \mathrm{~nm}$ (Region I) and the fractal dimension decreased with increasing scale length (Regions II \& III). Nevertheless, the fractal dimensions in all three regions were nearly two. This implies that the aspolished surfaces were quite smooth over the whole range of scales used for the calculation.

Figs. 2(c) and (d) exhibit the AFM images of the as-oxidized samples of 304 and 439 SS, respectively, formed in $5 \mathrm{wt} \% \mathrm{H}_{2} \mathrm{SO}_{4}$ solution. The two images looked quite different in terms of the degree of height fluctuation on a relatively small scale. The analysis of the scaling property of each surface clearly showed the difference (Fig. 3(b) and Table 1): The absolute slope, $\left|d l n A_{s c} / d l n L_{p s}\right|$, obtained from the surface of 439 SS was much larger (more than 10 times) than that of $304 \mathrm{SS}$, especially at a scale of less than ca. $30 \mathrm{~nm}$ (Region I'), causing a difference in the fractal dimension between the 439 and 304 SSs of approx. 0.02. On the other hand, the fractal dimensions at larger scales (Regions II' \& III') were estimated to be comparable to each other. The high irregularity at a scale of less than several tens of $\mathrm{nm}$ implies that the electrochemical reaction proceeds under quite different conditions from that of the perfectly smooth surface with two Euclidean dimensions, because the size order of the irregularity is in the mesopore regime where the Knudsen transport is dominant ${ }^{16,17)}$.

The origin of the difference in the surface morphology has yet to be determined and its detailed investigation is beyond the scope of this work. Nevertheless, the following two possibilities are most likely: One is that the tendency for uniform growth (or ordering of the atoms) of the oxide film on 304 SS is stronger 


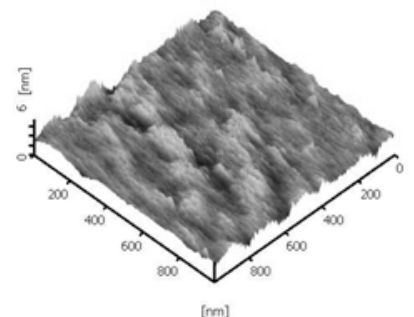

(a)

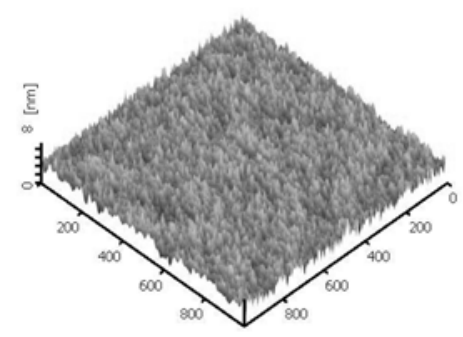

(d)

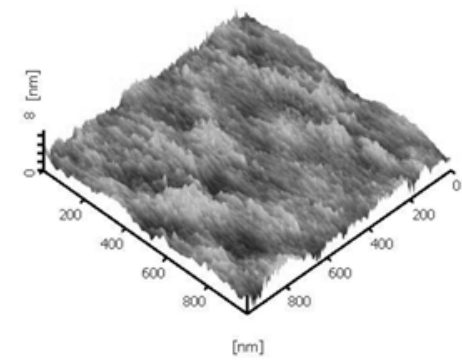

(b)

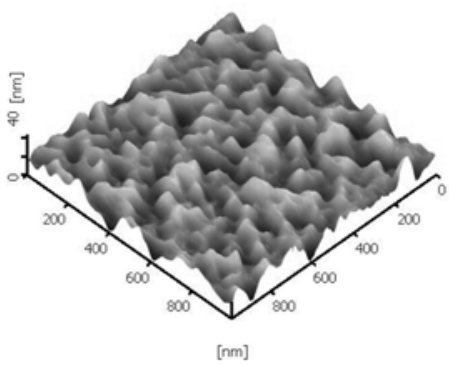

(e)

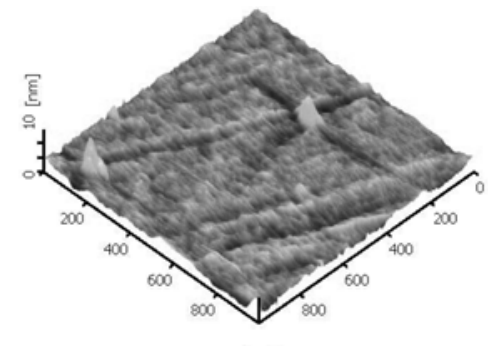

$[\mathrm{nm}]$

(c)

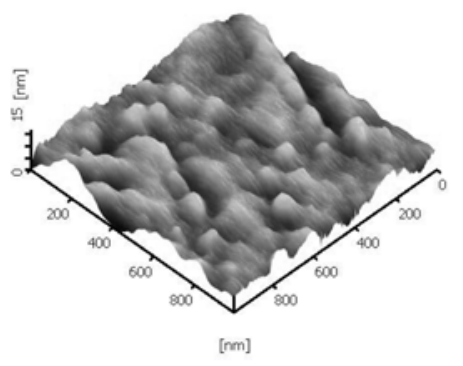

(f)

Fig. 2. AFM images of the SS surfaces. (a) and (b) are the images for the as-polished 304 and 439 SSs; (c) and (d) are the images for the as-oxidized 304 and $439 \mathrm{SSs}$ in $5 \mathrm{wt} \% \mathrm{H}_{2} \mathrm{SO}_{4}$, and (e) and (f) represent the images for the as-oxidized 304 and $439 \mathrm{SSs}$ in $5 \mathrm{wt} \% \mathrm{H}_{2} \mathrm{SO}_{4}+3.5 \mathrm{wt} \% \mathrm{NaCl}$ aqueous solution.

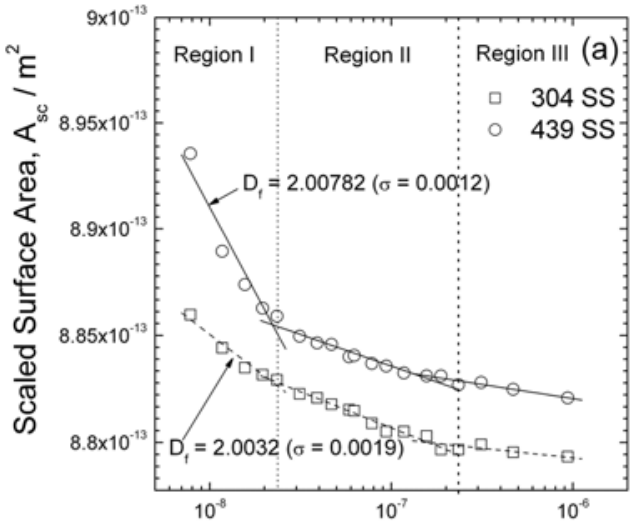

Projected Triangle Size, $\mathrm{L}_{\mathrm{ps}} / \mathrm{m}$

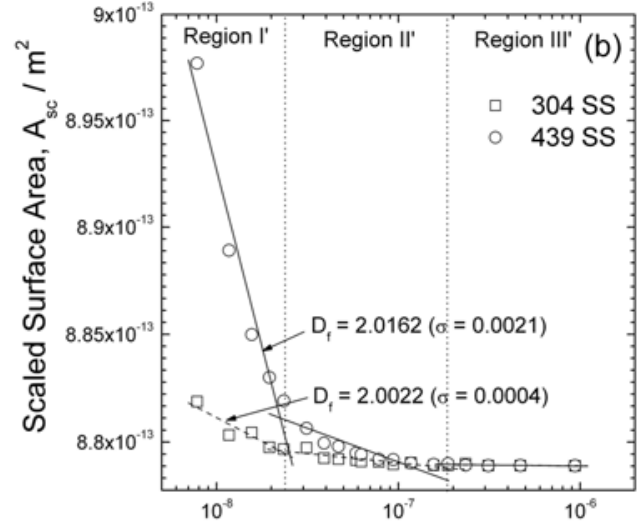

Projected Triangle Size, $\mathrm{L}_{\mathrm{ps}} / \mathrm{m}$

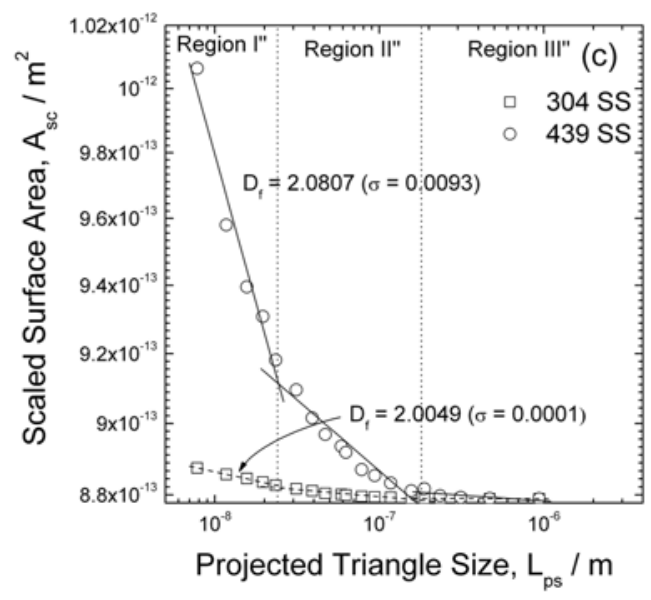

Fig. 3. Dependence of the scaled surface area $A_{s c}$ on the projected triangle size $L_{p s}$ for (a) the as-polished samples, (b) the as-oxidized samples in $5 \mathrm{wt} \% \mathrm{H}_{2} \mathrm{SO}_{4}$ solution and (c) the as-oxidized samples in $5 \mathrm{wt} \% \mathrm{H}_{2} \mathrm{SO}_{4}+3.5$ wt $\% \mathrm{NaCl}$ solution, determined from the triangulation analysis of Fig. 2. 
Table 1. The surface fractal dimensions $D_{f}$ of the passivated surface of 304 and $439 \mathrm{SSs}$ in $5 \mathrm{wt} \% \mathrm{H}_{2} \mathrm{SO}_{4}$ and 5 wt $\%$ $\mathrm{H}_{2} \mathrm{SO}_{4}+3.5 \mathrm{wt} \% \mathrm{NaCl}$ aqueous solutions, determined from the triangulation analysis of AFM images

\begin{tabular}{|c|c|c|c|c|c|c|c|c|c|c|}
\hline & \multicolumn{3}{|c|}{ As-polished } & \multicolumn{3}{c|}{$\begin{array}{c}\text { As-oxidized } \\
\left(5 \mathrm{wt} \% \mathrm{H}_{2} \mathrm{SO}_{4}\right)\end{array}$} & \multicolumn{3}{c|}{$\begin{array}{c}\text { As-oxidized } \\
\left(5 \mathrm{wt} \% \mathrm{H}_{2} \mathrm{SO}_{4}+3.5 \mathrm{wt} \% \mathrm{NaCl}\right)\end{array}$} \\
\cline { 2 - 9 } & Region I & Region II & Region III & Region I' & Region II' & Region III' & Region I"' & Region II" & Region III" \\
\cline { 2 - 10 } & \multicolumn{7}{|c|}{$D_{f}$ (standard deviation) from the AFM images } \\
\hline \multirow{2}{*}{$304 \mathrm{SS}$} & $\begin{array}{c}2.0032 \\
( \pm 0.0019)\end{array}$ & $\begin{array}{c}2.0026 \\
( \pm 0.0002)\end{array}$ & $\begin{array}{c}2.0004 \\
( \pm 0.0002)\end{array}$ & $\begin{array}{c}2.0022 \\
( \pm 0.0004)\end{array}$ & $\begin{array}{c}2.0004 \\
( \pm 0.0001)\end{array}$ & $\begin{array}{c}2.0001 \\
( \pm 0.0001)\end{array}$ & $\begin{array}{c}2.0049 \\
( \pm 0.0001)\end{array}$ & $\begin{array}{c}2.0019 \\
( \pm 0.0002)\end{array}$ & $\begin{array}{c}2.0001 \\
( \pm 0.0001)\end{array}$ \\
\hline 439 SS & $\begin{array}{c}2.0078 \\
( \pm 0.0012)\end{array}$ & $\begin{array}{c}2.0014 \\
( \pm 0.0001)\end{array}$ & $\begin{array}{c}2.0006 \\
( \pm 0.0001)\end{array}$ & $\begin{array}{c}2.0162 \\
( \pm 0.0021)\end{array}$ & $\begin{array}{c}2.0016 \\
( \pm 0.0002)\end{array}$ & $\begin{array}{c}2.0001 \\
( \pm 0.0001)\end{array}$ & $\begin{array}{c}2.0807 \\
( \pm 0.0093)\end{array}$ & $\begin{array}{c}2.0194 \\
( \pm 0.0020)\end{array}$ & $\begin{array}{c}2.0015 \\
( \pm 0.0007)\end{array}$ \\
\hline
\end{tabular}

than that on 439 SS, which makes the structure of the former oxide film more compact than that of the latter. This suggestion is consistent with the previous report that the crystallization of the oxide film on the austenitic SS proceeds faster than on the ferritic SS, due mainly to the enrichment of $\mathrm{Ni}$ in the metallic phase underneath the oxide film ${ }^{6}$. The low apparent density of the oxide film on 439 SS is not adequate to effectively protect the bare surface against the environment, since the porous nature of the oxide film is usually unfavorable to its passivity. In spite of the above arguments, however, considering that the thickness of the oxide film formed in an acid-based solution is typically less than $2 \mathrm{~nm}^{18)}$, factors other than the irregularity of the oxide film itself are needed to explain the relatively large height fluctuation of the surface morphology of 439 SS.

The other plausible factor is the rough character of the bare surface beneath the oxide film. The current density in the active nose region of $439 \mathrm{SS}$ is more than 2 orders of magnitude higher than that of 304 SS, as shown in Fig. 1(a). Accordingly, in spite of the instantaneous increase in the potential in the passive region employed to minimize the active dissolution of the metal (Fig. 1(a)), the change in the morphology of the bare surface is unavoidable in the course of oxide formation. On the other hand, the irregular/rough bare surface is most likely detrimental to the corrosion resistance of the SS, because there is great possibility for large amounts of defects (e.g., grain boundaries, flaws, voids and pores) to be included in the oxide film when it is formed on the highly-irregular bare surface. At the same time, an irregular surface with a large area leads to the rise in the apparent electrochemical activity, which additionally increases the metal dissolution rate.

Based on the above argumentation, it is conceivable that the lower surface irregularity of the SS, especially at a scale of less than several tens of nanometers is quite beneficial to its resistance against a corrosive environment. Also, to estimate the irregularity of the surface, the self-similar fractal dimension based on the scaling properties of the surface morphology can be effectively utilized and might give useful information on the actual protectiveness of the oxide film.

Shown in Figs. 2(e) and (f) are the AFM images of the as-oxidized samples of 304 and $439 \mathrm{SSs}$, respectively, formed in $5 \mathrm{wt} \% \mathrm{H}_{2} \mathrm{SO}_{4}+3.5 \mathrm{wt} \% \mathrm{NaCl}$ solution. The vertical fluctuations of their surface heights are much larger than those of the samples formed in pure acid solution (Fig. 2(c) and (d)) (please note the scale of the vertical axis), which resulted in the larger fractal dimensions of the samples oxidized in chloride-containing acid solution, as compared to those in pure acid solution (Fig. 3(c) and Table 1). In particular, it is remarkable that there is a significant difference in the fractal dimension of the as-oxidized $439 \mathrm{SS}$ according to the inclusion or not of chloride ions in the passivating solution. This strongly indicates that the chloride ions severely interfere with the uniform corrosion of the bare surface and/or the uniformity of the oxide film and, furthermore, that the $439 \mathrm{SS}$ is much more susceptible to local damage induced by the chloride ions than the $304 \mathrm{SS}$.

\section{Conclusions}

An in-depth morphological investigation of the surface of passivated 304 and 439 SSs was conducted on the basis of the scaling property. The self-similar fractal dimensions of the irregular surface were estimated over the range of about three orders of magnitude range between $2 \mathrm{~nm}$ and $500 \mathrm{~nm}$. The surface morphology of the passivated 439 SS formed in chloride-free and chloride-containing acid solutions showed a higher fractal dimension than that of the passivated 304 SS, particularly at a scale of several tens of nanometers, strongly indicating the relatively high irregularity in the surface of the passivated 439 
SS. It is expected that the self-similar fractal dimension could be effectively utilized for estimating the irregularity of the passivated surface and could provide us with useful information on the actual protectiveness of the oxide film.

\section{Acknowledgements}

This work was supported for two years by Pusan National University Research Grant.

\section{References}

1. P. Schmuki, J. Solid State Electrochem., 6 (2002) 145.

2. C.-O. A. Olsson, D. Landolt, Electrochim. Acta, 48 (2003) 1093.

3. C. Calinski, H.-H. Strehblow, J. Electrochem. Soc., 136 (1989) 1328.

4. M. F. Toney, A. J. Davenport, L. J. Oblonsky, M. P. Ryan, C. M. Vitus, Phys. Rev. Lett., 79 (1997) 4282.

5. P. Schmuki, S. Virtanen, H. S. Isaacs, M. P. Ryan, A. J. Davenport, H. Böhni, T. Stenberg, J. Electrochem. Soc., 145 (1998) 791.
6. V. Maurice, W. P. Yang, P. Marcus, J. Electrochem. Soc., 145 (1998) 909.

7. D. Zuili, V. Maurice, P. Marcus, J. Phys. Chem. B, 103 (1999) 7896.

8. B. B. Mandelbrot, The Fractal Geometry of Nature, Freeman, New York, 1983.

9. B. B. Mandelbrot, D. E. Passoja, A. J. Paullay, Nature, 308 (1984) 721.

10. C. S. Pande, L. E. Richards, N. Louat, B. D. Dempsey, A. J. Schwoeble, Acta Metall., 35 (1987) 1633.

11. Z. G. Wang, D. L. Chen, X. X. Jiang, S. H. Ai, C. H. Shih, Scripta Metall., 22 (1988) 827.

12. L. Nyikos, T. Pajkossy, Electrochim. Acta, 30 (1985) 1533.

13. B. Sapoval, J.-N. Chazalviel, J. Peyriere, Phys. Rev. A, 38 (1988) 5867.

14. C. Douketis, Z. Wang, T. L. Haslett, M. Moskovits, Phys. Rev. B, 51 (1995) 11022.

15. H.-C. Shin, S.-I. Pyun, J. Electroanal. Chem., 531 (2002) 101.

16. M. Knudsen, Ann. Phys. (Leipzig), 28 (1909) 75.

17. A.J. Burggraaf, J. Membr. Sci., 155 (1999) 45.

18. S. Haupt, H.-H. Strehblow, Corros. Sci., 37 (1995) 43. 